\title{
ARTICLE \\ Association of childhood traumatization and neuropsychiatric outcomes with altered plasma micro RNA-levels
}

\author{
Sandra Van der Auwera ${ }^{1,2}$, Sabine Ameling ${ }^{3,4}$, Katharina Wittfeld iD $^{1,2}$, Enrique d'Harcourt Rowold ${ }^{1}$, Matthias Nauck ${ }^{4,5}$, \\ Henry Völzke ${ }^{4,6}$, Karsten Suhre ${ }^{7}$, Hani Najafi-Shoushtari ${ }^{8,9}$, Jaicy Methew ${ }^{9}$, Vimal Ramachandran ${ }^{9}$, Robin Bülow $^{10}$, \\ Uwe Völker (iD ${ }^{3,4}$ and Hans J. Grabe (iD ${ }^{1,2}$
}

\begin{abstract}
Childhood traumatization (CT) is associated with the development of several neuropsychiatric disorders in later life. Experimental data in animals and observational data in humans revealed evidence for biological alterations in response to CT that may contribute to its long-term consequences. This includes epigenetic changes in miRNA levels that contribute to complex alterations of gene expression. We investigated the association between CT and 121 miRNAs in a target sample of $N=150$ subjects from the general population and patients from the Department of Psychiatry. We hypothesized that CT exhibits a long-term effect on miRNA plasma levels. We supported our findings using bioinformatics tools and databases. Among the 121 miRNAs 22 were nominally significantly associated with CT and four of them (let-7g-5p, miR-103a-3p, miR-107, and miR-142-3p) also after correction for multiple testing; most of them were previously associated with Alzheimer's disease (AD) or depression. Pathway analyses of target genes identified significant pathways involved in neurodevelopment, inflammation and intracellular transduction signaling. In an independent general population sample $(N=587)$ three of the four miRNAs were replicated. Extended analyses in the general population sample only $(N=687)$ showed associations of the four miRNAs with gender, memory, and brain volumes. We gained increasing evidence for a link between $C T$, depression and $A D$ through miRNA alterations. We hypothesize that depression and $A D$ not only share environmental factors like CT but also biological factors like altered miRNA levels. This miRNA pattern could serve as mediating factor on the biological path from CT to adult neuropsychiatric disorders.
\end{abstract}

Neuropsychopharmacology (2019) 44:2030-2037; https://doi.org/10.1038/s41386-019-0460-2

\section{INTRODUCTION}

Childhood traumatization $(\mathrm{CT})$ is associated with the development of several mental and neurological disorders in later life, such as depression, anxiety, or cognitive impairment [1].

Experimental data in animal models and observational data in humans have accumulated evidence for biological alterations in response to $\mathrm{CT}$ that may contribute to long-term consequences [2-4]. Nevertheless, the understanding of the complex regulatory mechanisms is still limited.

Recently, it has been discovered that the long-lasting effects of CT may also affect gene expression $[5,6]$. These changes demonstrate the impact of the environment on genes and their regulation. These epigenetic alterations do not change the genetic code, but rather influence the regulation of gene expression and enable the individual to respond quickly to environmental challenges. Epigenetic regulators as micro RNAs (miRNA) are small ( $\approx 22 \mathrm{nt}$ ) noncoding RNAs which play important regulatory roles by targeting mRNAs for degradation, mediating translational repression or in rare cases enhance transcription by binding to the promoter region of target genes [7, 8]. Gene regulation by miRNAs is far-reaching as one miRNA can interact with many hundreds of mRNAs and one mRNA can be targeted by several miRNAs [9].

Regarding traumatic events and CT, there is increasing evidence of alterations in miRNA profiles. First studies regarding the longterm effect of early trauma have been performed using animal models of mice $[4,10,11]$. Gapp et al. showed that altered miRNA profiles in sperm induced via unpredictable separation of young male mice from their mother can be transferred into the next generation [4]. After separation from their mother, these mice developed a depressive-like phenotype as well as altered miRNA profiles compared with control mice. These changes were also visible in the next two generations, even if the progeny has not been exposed to stress. Analyses of miRNA profiles showed that they were transferred via sperm into the next generation. In young female mice, Kumari et al. found that after social isolation the expression of BDNF-associated miRNAs was altered and BDNF

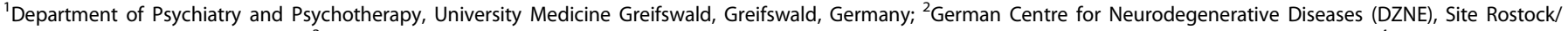

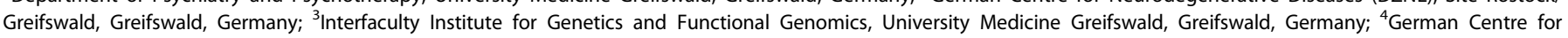

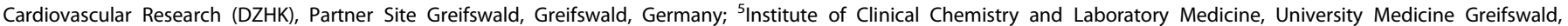

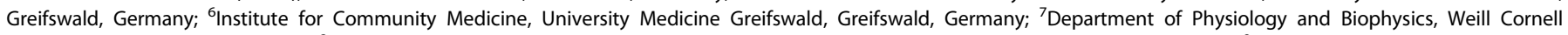

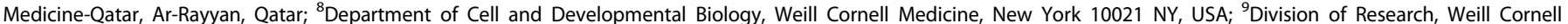

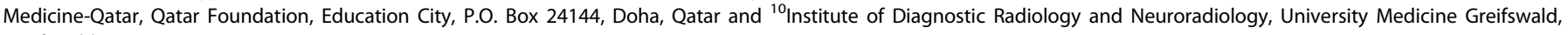
Greifswald, Germany

Correspondence: Sandra Van der Auwera (auweras@uni-greifswald.de)

These authors contributed equally: Sandra Van der Auwera, Sabine Ameling, Uwe Völker and Hans J. Grabe

Received: 18 January 2019 Revised: 27 June 2019 Accepted: 28 June 2019

Published online: 8 July 2019 
Table 1. Sample characteristic of clinical sample (GANI_MED) and general population sample (SHIP-TREND)

\begin{tabular}{|c|c|c|c|c|}
\hline Variable & GANI_MED $(N=50)$ & $\begin{array}{l}\text { SHIP-TREND } \\
(N=100)\end{array}$ & Comparison & SHIP-TREND full $(N=687)$ \\
\hline $\operatorname{Sex}(\%)$ & $\begin{array}{l}\text { Males: } 14(28 \%) \\
\text { Females: } 36(72 \%)\end{array}$ & $\begin{array}{l}\text { Males: } 66(66 \%) \\
\text { Females: } 34(34 \%)\end{array}$ & $\begin{array}{l}\text { Chi2 }=19.34 \\
P<0.001\end{array}$ & $\begin{array}{l}\text { Males: } 348(50.7 \%) \\
\text { Females: } 339(49.3 \%)\end{array}$ \\
\hline Age (Mean(sd), range) & $\begin{array}{l}37.5(13.1) \\
{[19-63]}\end{array}$ & $\begin{array}{l}38.7(11.2) \\
{[21-63]}\end{array}$ & $\begin{array}{l}T=0.57 \\
P=0.57\end{array}$ & $\begin{array}{l}49.9(13.7) \\
{[21,79]}\end{array}$ \\
\hline BMI (Mean(sd), range) & $\begin{array}{l}29.4(6.4) \\
{[15.94,43.17]}\end{array}$ & $\begin{array}{l}26.4(3.9) \\
{[19.34-37.97]}\end{array}$ & $\begin{array}{l}T=-3.50 \\
P<0.001\end{array}$ & $\begin{array}{l}27.4(4.3) \\
{[17.74,48.05]}\end{array}$ \\
\hline PHQ-9 (Mean(sd), range) & $\begin{array}{l}27(4.6) \\
{[9-23]}\end{array}$ & $\begin{array}{l}13(3.4) \\
{[15-36]}\end{array}$ & $\begin{array}{l}T=-20.80 \\
P<0.001\end{array}$ & $\begin{array}{l}12.8(3.5) \\
{[9,35]}\end{array}$ \\
\hline Hematocrit (Mean(sd)) & $0.42(0.04)$ & $0.43(0.03)$ & $T=0.57, P=0.57$ & $0.42(0.03)$ \\
\hline Platelet count (Mean(sd)) & $283(73)$ & $223(47)$ & $T=-6.12, P<0.001$ & $226(49)$ \\
\hline NAI (immediate recall), mean(sd), range & NA & NA & NA & $5.4(1.2),[0,8]$ \\
\hline Bilateral hippocampus volume $\left(\mathrm{cm}^{3}\right)$ & NA & NA & NA & $80(9),[51,110]$ \\
\hline
\end{tabular}

expression was upregulated [10]. Also these mice exhibited an anxiety phenotype. Recently, Dickson et al. tried to transfer these results to men [11]. They compared sperm miRNA changes in mice and men who had been exposed to early life stress and found several miRNAs differentially expressed. Although only $N=28$ men participated, this study conveys strong evidence that this type of epigenetic regulation after CT exists and that it could play a role in the transgenerational transmission of the vulnerability to mental disorders. Cattane et al. identified one miRNA associated with early life stress across different human and animal tissues [6] with a potential relevance in schizophrenia. But again with a very small target sample of only $N=32$ subjects.

miRNAs are not only present intracellularly but also in different body fluids, particularly in blood. Due to their high ribonuclease resistance circulating miRNAs are easily detectable. In our study, we want to confirm whether CT leaves an epigenetic "fingerprint" visible as alterations in miRNA plasma levels. We investigated the association between $\mathrm{CT}$ and miRNA in subjects from two different settings, $N=100$ subjects from the general population and $N=50$ patients from the Department of Psychiatry of the University Medicine Greifswald. We hypothesized that CT exhibits a longterm effect on miRNA plasma pattern and that these miRNAs target specific genes and biological pathways involved in gene regulation, especially in the CNS. Replication of the most significant results was performed in a larger general population sample $(N=587)$.

\section{MATERIALS AND METHODS}

Ethics statement

The study has been conducted to the recommendations of the Declaration of Helsinki. The Study protocol of SHIP-TREND [12] and GANI_MED [13] was approved by the medical ethics committee of the University of Greifswald. Written informed consent was obtained from each of the study participants.

Study design

GANI_MED is a multidisciplinary clinical cohort $(N>4500)$ enrolling patients with metabolic, cardiovascular, neurological diseases, and mental disorders. For our micro RNA analyses we only included participants with mental disorders. EDTA-plasma samples from $N=50$ GANI_MED participants from the Department of Psychiatry with the highest scores for CT were selected $(N=14$ males, $N=36$ females).

SHIP-TREND is a general population cohort from the Study of Health in Pomerania drawn from 2008 to 2012 with the aim to assess the prevalence and incidence of common diseases and their risk factors in the population. $N=100$ participants (out of the full miRNA SHIP-TREND sample, $N=687$ ) were selected matching in age with GANI_MED participants (see Supplemental Material). To ensure the balance between males and females in combined analyses, we selected $N=66$ males and $N=34$ females. The descriptive statistics of the included GANI_MED and SHIP-TREND participants is given in Table 1.

Detailed information on both cohorts can be found elsewhere $[12,13]$. A list with mental and cardiovascular diagnoses of the $N=$ 50 GANI_MED and the $N=100$ SHIP-TREND-0 participants is given in the supplement.

\section{Phenotype measures}

Current depressive symptoms were assessed using the 9-item sum score of the self-report Patient Health Questionnaire (PHQ-9) [14].

In SHIP-TREND the Childhood Trauma Questionnaire (CTQ) was used for self-report of CT [15]. The CTQ has 34 items that were rated on a five-point Likert scale with higher scores indicating more self-rated exposure to traumatic events. It covers the five subdimensions emotional, physical, and sexual abuse as well as emotional and physical neglect. In GANI_MED the validated 5-item CTQ screener (CTS) [16] was used with one question for each of the five sub-dimensions rated on a fivepoint Likert scale. As these five questions are also part of the $\mathrm{CTQ}$, in both cohorts we calculated an overall trauma score as the sum of the five CTS questions covering all five subdimensions of abuse and neglect.

In SHIP-TREND, the word list of the Nuremberg Age Inventory (NAl) was used to assess the declarative memory (immediate and delayed recall of neutral words) [17]. Detailed information on the NAI is given in the supplement. Subjects from SHIPTREND were asked to participate in a whole-body magnetic resonance imaging assessment [3]. Data on total gray matter and hippocampus volume read-out were available for $N=647$ and $N=622$ subjects, respectively. A detailed description on 
Discovery

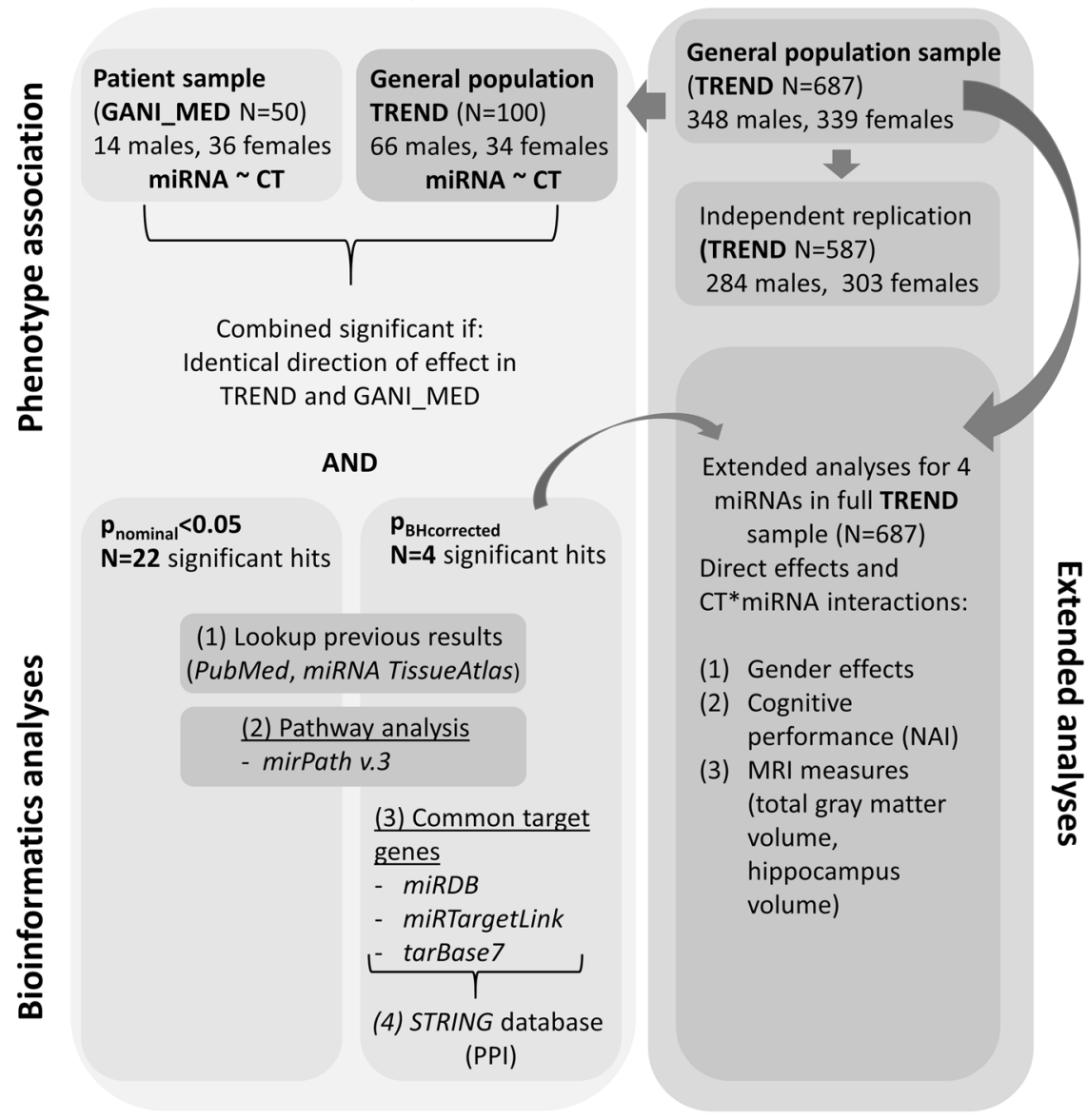

Fig. 1 Workflow of statistical, bioinformatics, and extended analyses

exclusion criteria, data preprocessing and volume read-out can be found in the supplement.

\section{Plasma miRNAs}

Noncellular blood circulating miRNAs were profiled as described previously [18]. Briefly, noncellular blood circulating miRNAs were prepared from $200 \mu \mathrm{l}$ EDTA plasma using the miRCURY' ${ }^{\mathrm{TM}}$ RNA Isolation Kit -Biofluids (Qiagen, Hilden, Germany). For RT-qPCRbased miRNA analysis, the Serum/Plasma Focus microRNA PCR Panel V4.M (Qiagen, Hilden, Germany) was used, covering 179 miRNAs. Details on profiling and quality control as well as on the preprocessing of qRT-PCR data are provided in the supplement.

\section{Phenotype association}

miRNA residuals after adjustment for technical variables (see supplement) were used as dependent variables. As independent variable, the CTS was used. Primary analyses were conducted in each cohort separately adjusted for a limited set of covariates (age, sex, PHQ-9) because of small sample sizes. Combined analyses were also adjusted for cohort. Sensitivity analyses were performed with a second set of covariates including BMI and blood cell parameters (hematocrit, platelet count). All confidence intervals and $p$-values were obtained from bootstrapping with $N=1000$ replications to account for small sample sizes. Results were assumed to be statistically significant if the direction of the sign of the regression coefficient was identical in SHIP-TREND and GANI_MED and if the combined analysis of SHIP-TREND + GANI_MED was significant with $p_{\text {nominal }}<0.05$. All reported $p$-values are two-sided. The phenotype association analyses have been performed with STATA 14 [19].
Permutation analysis

To confirm the statistical significance of our results, we created $N=100$ permutations of the CTS phenotype and repeated the association analyses. For every permutated phenotype we stored the number of significant results. A mean \pm sd of $6.8 \pm 3.9 \%$ of the miRNAs was significant for each permutated phenotype (median $5.8 \%$, $\min .0 .08 \%$, max. $28 \%$ ).

\section{Bioinformatics analyses}

For most significant results, we performed a bioinformatics analysis using publically available resources. (1) Lookup of previous results in PubMed; (2) looking for enrichments in predefined pathways using DIANA tools mirPath v.3 [20] where miRNAs are taken as input to identify target genes and among them search for enrichment within the KEGG pathway database; (3) identification of common target genes in three human miRNA target gene databases (miRDB http://www. mirdb.org/ [21], miRTargetLink https://ccb-web.cs.uni-saarland. de/mirtargetlink/ [22], and tarBase7 http://diana.imis.athenainnovation.gr/DianaTools/index.php?r=tarbase/index [23]); 4. protein-protein interaction networks of target gene sets using the STRING database [24].

Extended analyses in general population sample (SHIP-TREND) Within the remaining subjects from SHIP-TREND $(N=587$, excluding $N=100$ from the target sample), we performed independent replication of significant miRNAs. Within the full SHIP-TREND sample comprising $N=687$ subjects, we performed extended analyses for the significant miRNAs. (1) Analysis of gender effects; and analyses using additional phenotypes, (2) 


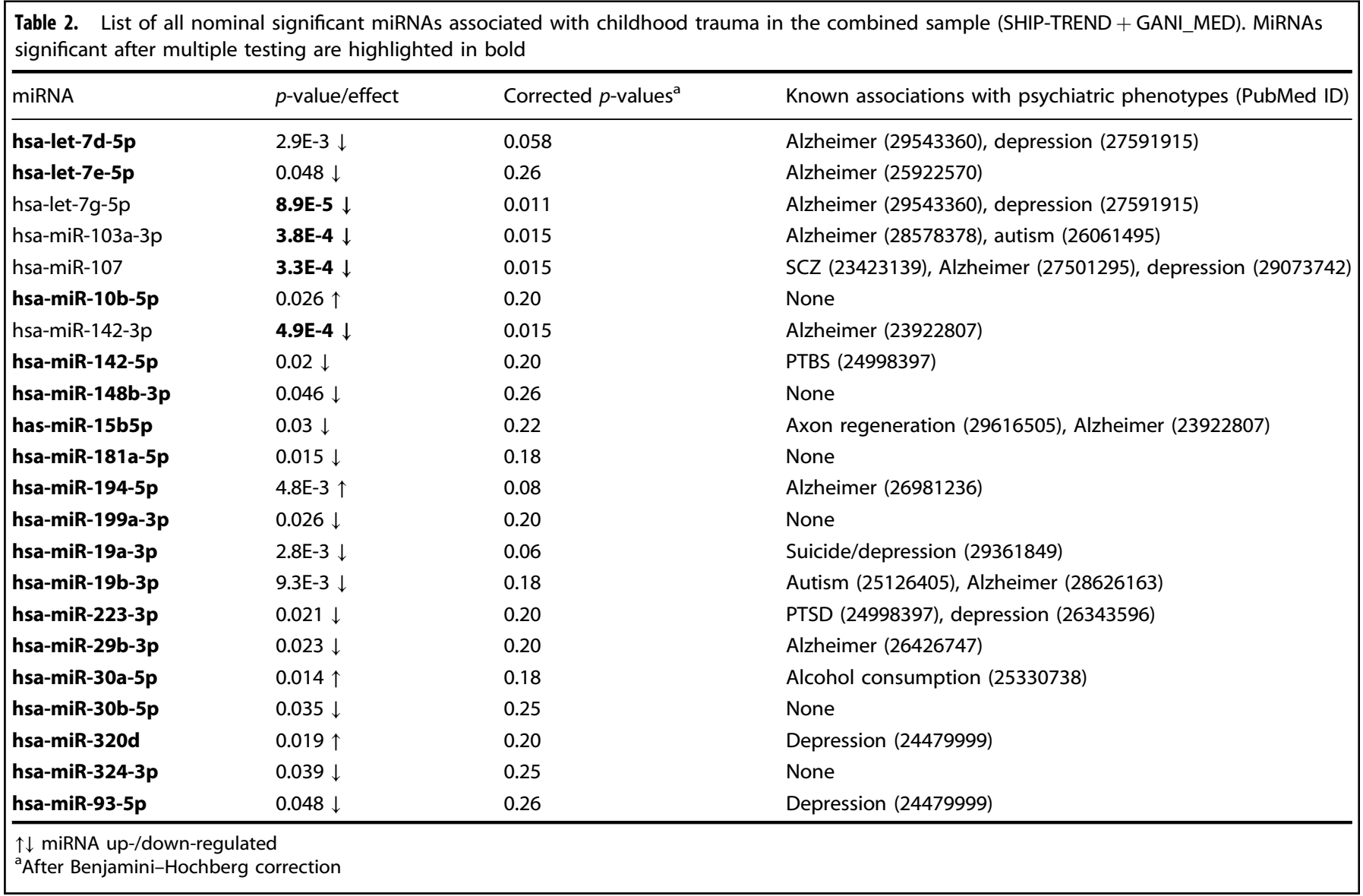

cognitive performance, and (3) MRI measures (total gray matter volume, hippocampus volume).

For a graphical overview of the phenotype associations and bioinformatics workflow see Fig. 1.

\section{RESULTS}

A brief sample characteristic is given in Table 1 . We excluded miRNAs that were not detectable in $>50 \%$ of the subjects of each sample leaving a set of $N=121$ miRNAs for the final association test (full list see supplement).

Of the 121 miRNAs $N=22(18 \%)$ were associated with CTS in the combined sample according to our significance criteria (for the full results of the two discovery samples and the combined sample see supplementary Table S1). Four of these 22 miRNAs showed higher and 18 lower levels with increased CTS. After Benjamini-Hochberg correction for multiple testing, $N=4$ miRNAs were still associated with CTS (Table 2, Fig. 2; hsa-let7g-5p, hsa-miR-103a-3p, hsa-miR-107, and hsa-miR-142-3p). In the following subsequent bioinformatics investigations, we focused on those four miRNAs and extended this analysis in the general population sample. The pairwise correlation between the four miRNAs was moderate from $r=0.47$ (hsa-let7g-5p hsa-miR-107) to $r=0.73$ (hsa-miR-103a-3p hsa-miR-1423p) (see Table S2).

Compared with our permutation analysis, our result of $18 \%$ significant miRNAs is about three standard deviations above the mean and thus highly significant. Sensitivity analysis including BMI and blood cell parameters did not change the significance of the results. A scatter plot of the linear association between $C T$ and the four miRNAs is given in Fig. S1 in the supplement.
Biological insight of significant miRNAs

The four miRNAs belong to three miRNA families; hsa-mir-103 (hsa-miR-103a-3p, hsa-miR-107), hsa-mir-142 (hsa-miR-142-3p), and hsa-let-7 (hsa-let-7g-5p) [25]. Except for hsa-miR-142-3p, they are broadly expressed in the brain according to the miRNA Tissue Atlas (https://ccb-web.cs.uni-saarland.de/tissueatlas/patterns) [25] where data from 1997 miRNAs in 61 tissues are stored (Fig. S2). Hsa-let-7g-5p is located on chromosome 3 within the WDR82 gene. It has been associated with Alzheimer's disease (AD) and major depression [26-28]. Hsa-miR-103a-3p is located on chromosome 20 lying within the PANK2 gene. The miRNA product has been associated with $A D$ [28] and autism [29]. Hsa-miR-107 is located on chromosome 10 within the PANK1 gene. This miRNA transcript is homolog to hsa-miR-103 and has been associated with the circadian system [30]. On the neuropsychiatric level hsamiR-107 has been associated with major depression [7], schizophrenia [31] and AD [32]. One study found BDNF, a longstanding candidate gene for mental disorders, to be a target of hsa-miR-107 [33]. Hsa-miR-142-3p is located on chromosome 17 within TSPOAP1-AS1. Like the three miRNAs before, hsa-miR-142-3p has been found to be associated with AD [34].

Pathway analysis

Pathways were identified using mirPath v.3. As three different methods are available to assign target genes to miRNAs (TargetScan database, Tarbase database, and microT-CDS algorithm) [20], we performed all of them and sorted the results according to their combined sum rank. A detailed description of these methods is given in the supplement. We only report pathways that were significant $(p<0.05)$ in all approaches. Taking the four confirmed top miRNAs as input, $N=8$ pathways emerged 


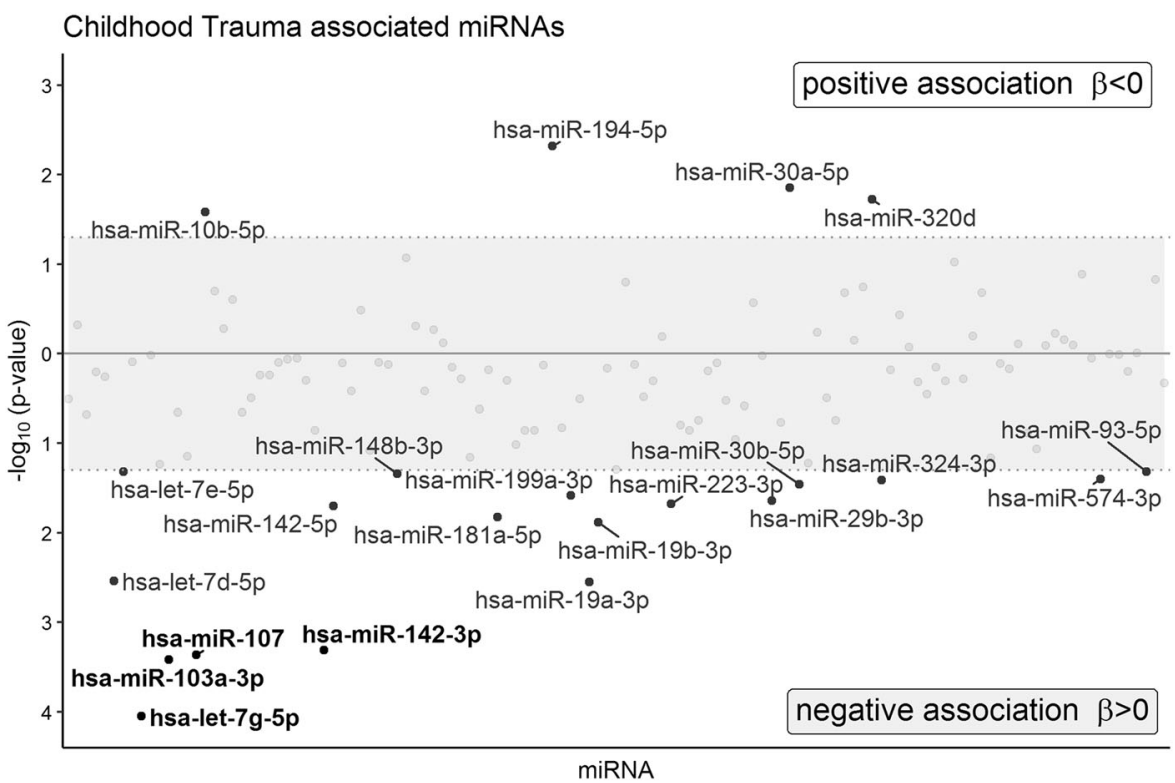

Fig. 2 Manhattan-like plot displaying $p$-values and directions of effect for the association results of $N=121$ miRNAs with childhood trauma score in the combined sample (SHIP-TREND + GANI_MED). All at least nominal significant miRNAs are listed. miRNAs significant after Benjamini-Hochberg correction are bolt

as significant. We excluded pathways associated with special nonmental diseases which are mostly the variations of other signaling pathways (e.g., cancer pathways). The remaining $N=5$ pathways were fatty acid metabolism, mTOR signaling (involved in neurodevelopment), MAPK signaling (intracellular transduction signaling), regulation of actin cytoskeleton, and signaling pathways regulating pluripotency of stem cells.

Taking all $N=22$ nominally significant miRNAs as input, 54 pathways were significantly influenced. Excluding pathways associated with special nonmental diseases, $N=35$ pathways remained which were involved in (1) neurodevelopment (e.g., Hippo signaling, FoxO signaling, mTOR signaling, and Wnt signaling), (2) inflammation (e.g., TGF-beta signaling, prolactin signaling), or (3) intracellular transduction signaling (e.g., PI3k-Akt signaling, MAPK signaling, neurotrophin signaling) [6]. Other interesting pathways were fatty acid biosynthesis, circadian rhythm, or long-term depression. For full results see supplementary Tables S3 and S4.

Common target genes

To get a deeper mechanistic insight of the biological role of the four miRNAs, we searched for common target genes using miRDB, miRTargetLink, and tarBase7. Target genes for a specific miRNA were defined as valid if they appeared in all three databases (see Fig. S3) leaving $N=73$ target genes for hsa-let-7g-5p, $N=95$ for hsa-miR-103a-3p, $N=75$ for hsa-miR-107, and $N=55$ for hsa-miR142-3p (Table S5). In the next step, we searched for target gene overlap between them. $N=75$ genes appeared to be a target gene for more than one miRNA with TGFBR3 (transforming growth factor beta receptor 3) being a target gene for hsa-miR-103a-3p, hsa-miR-107, and hsa-miR-142-3p. Results for the application of a more conservative evidence score of the databases can be found in the supplement.

\section{Interaction networks}

With one target gene set for each miRNA we received four protein interaction networks (supplementary Figs. S4-S7) using the STRING database. As it can be seen, the networks of hsa-miR103a-3p, hsa-miR-107, and hsa-let-7g-5p show a stronger interaction pattern than the network for hsa-miR-142-3p. Supplementary
Table S6 lists all proteins with at least three interaction partners in the networks representing proteins regulating critical points of gene regulation.

Independent replication in the general population (SHIP-TREND $N=587$ )

Using the remaining subjects of the SHIP-TREND miRNA sample (excluding the target sample $N=100$ ), we performed independent replication in $N=587$ subject $(N=284$ males, $N=303$ females). As the range of the CTS was significantly lower than in the clinical sample, we created a dichotomous CT phenotype with low CTS values $(5-6 ; N=367)$ versus high CTS values $(>6 ; N=$ 223). Linear regression analyses were performed with dichotomous CT as predictor and miRNA as outcome (adjusted for age, sex, PHQ-9, hematocrit, BMI, and platelet count). We were able to replicate significant results of three miRNAs (hsa-miR-103a-3p $p=$ 6.4E-3, hsa-miR-107 $p=0.047$, hsa-miR-142-3p $p=7.7 \mathrm{E}-3$ ). Using the dimensional CTS score, only hsa-miR-103a-3p and hsamiR-107 could be replicated.

Extended analyses in the general population sample (SHIP-TREND, $N=687$ )

Extended analyses have been performed for the four significant miRNAs in the full SHIP-TREND miRNA sample (all adjusted for age, sex, PHQ-9, BMI, hematocrit, and platelet count).

1. Gender effects. As many miRNAs exhibit gender effects [18], we performed sex stratified analyses $(N=348$ males, $N=339$ females). Given the greater sample with equal balanced sex groups the results showed that the effects of CTS score on all four miRNAs were predominantly observed in males (supplementary Table S7).

2. Cognitive outcomes. Because all four significant miRNAs have previously been associated with $A D$ or cognition, we analyzed the direct association of NAl verbal memory scores (immediate and delayed recall) on miRNA as well as interactions with CTS. Two miRNAs revealed nominal significant effects for the interaction of immediate recall of words and CTS (hsa-miR-107, $p_{\mathrm{INT}}=0.049$; hsamiR-142-3p, $p_{\text {INT }}=0.032$ ). 

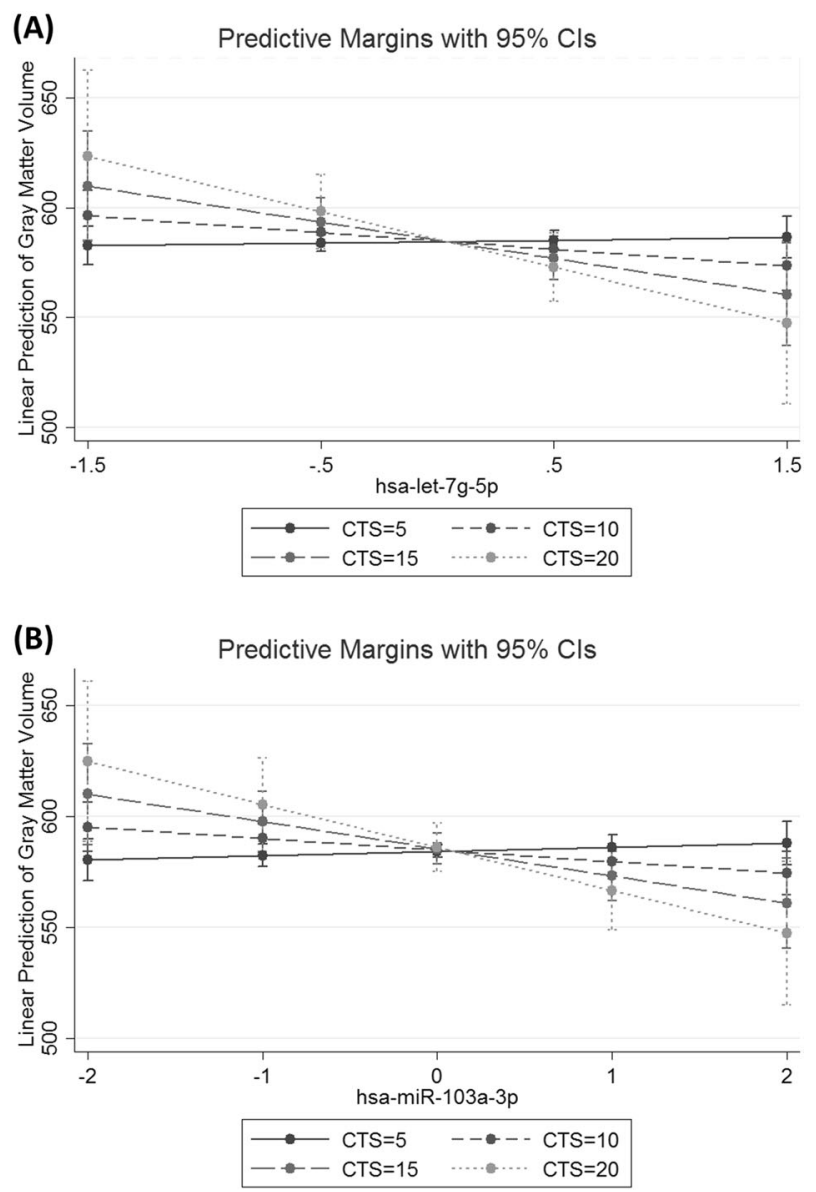

Fig. 3 Interaction plots for the significant interactions between CTS and miRNA on gray matter volume in the general population sample SHIP-TREND. a Interaction between CTS groups and has-let$7 g-5 p$ on gray matter volume. b Interaction between CTS groups and has-miR-103a-3p on gray matter volume

3. Brain imaging phenotypes. We analyzed the effect of the four miRNAs, as well as miRNA*CTS interaction on total gray matter and hippocampus volume (additionally adjusted for age $^{2}$ and intracranial volume). Again, for two miRNAs we exhibited nominal significant interaction effects (hsa-let-7g-5p, $p_{\text {INT }}=0.045$; hsa-miR$103 a-3 p, p_{\text {INT }}=0.028$ ) with higher CTS values plus lower miRNA levels associated with reduced gray matter volume. Regarding hippocampus volume only the hsa-miR-103a-3p*CTS interaction was nominal significant with $p_{\mathrm{INT}}=0.019$.

Interaction plots for gray matter results can be found in Fig. 3 and interaction plots for verbal memory can be found in the supplement (Fig. S8). In all extended analyses 1-3, additional adjustment for the lifestyle factors, such as current smoking, physical activity, and alcohol consumption did not change the significance of the results.

\section{DISCUSSION}

This is the first study investigating the long-term changes of plasma miRNA levels in association with $\mathrm{CT}$ in the general population and a clinical sample. We identified four miRNAs significantly associated with CT (hsa-let-7g-5p, hsa-miR-103a-3p, hsa-miR-107, and hsa-miR-142-3p). Previous studies searching for miRNA signatures associated to early life stress identified several miRNAs in small samples of at most $N=32$ subjects $[6,11]$. We were not able to replicate these miRNAs as they were not present in our miRNA panel. Our target sample comprises subjects from the general population $(N=100)$ and the Department of Psychiatry $(N=50)$. By that, we could test the effects of $C T$ on miRNA in different settings and thus improve specificity and generalizability of our findings. Moreover, we could test nonemoderate traumatized subjects from the general population in extended analyses $(N=687)$ using proxy phenotypes for AD.

As our findings suggest, $C T$ is associated with epigenetic changes in plasma levels of at least four miRNAs; all of them previously associated with $A D$ [27, 28, 34]. Many studies have shown that traumatization in early life is associated with decreased memory performance [35], structural and functional brain abnormalities [36] and even AD [37]. In SHIP-TREND we previously found that a higher childhood trauma load was associated with negative effects on verbal memory recall (Terock et al., submitted). In extended analyses in the general population two of the miRNAs were also associated with verbal memory and two with gray matter or hippocampus volume. This raises the hypothesis that the effect of childhood trauma on neurocognitive outcomes may be mediated through miRNAs.

Bioinformatics analyses support the association to AD. Of the $N=22$ nominally significant miRNAs $N=10$ have previously been associated with $A D$ with three of them belonging to the neurotoxic let7 miRNA family [38]. Pathway analyses showed that many pathways regulated by target genes of the significant miRNAs were involved in neurodevelopment, inflammation, intracellular transduction signaling, depression or fatty acid metabolism; pathways important for CNS functioning and associated with $A D[39,40]$. Analyses of common target genes identified many genes associated with neuropsychiatric phenotypes including TGFBR3 (transforming growth factor beta receptor $3)$, a receptor of TGF- $\beta$ s being key factors for the development of $\mathrm{AD}[41]$.

In extended analyses we were able to test the relevance of four miRNAs on AD proxy phenotypes. This includes sex differences which revealed a higher impact of the four miRNAs in males. On the genetic level such sex differences in the susceptibility of $A D$ and cognition have been identified [42-44]. Further, we found interaction effects with childhood trauma for the four miRNAs on immediate verbal memory recall as well as on total gray matter and hippocampus volume. Unfortunately, mediation effects of miRNA levels on the path from $C T$ to gray matter of verbal memory were not found which could also be a problem of still too small sample size. Also it has to be emphasized that SHIP-TREND is a non-dementia sample where it is even more difficult to detect effects of our AD proxies.

Changes in miRNA are also associated with depression [45]. One study investigating the shared biological pathways between $A D$ and depression based on miRNA expression identified 7 miRNAs being abnormally expressed in both disorders [26] providing a link between neurodegenerative and psychiatric disorders [46]. Two of them (has-let-7g-5p, hsa-let-7d-5p) were nominally associated with CT in our analyses and two (has-miR-191-5p, hsa-miR-26b-5p; data not shown) were nominally associated only in the GANI_MED sample. As also seven of the 22 nominal significant miRNAs have previously been associated with depression, a possible involvement of depressive disorders in the interplay between $\mathrm{CT}$, miRNA changes, and AD cannot be fully precluded. The development of depressive disorders in adulthood is strongly associated with childhood traumatic experiences [47]. Late life depression itself is a common co-occurrence for all forms of dementia [48]. This raises the question if $A D$ and depression share not only common environmental factors but also biological factors. On the genetic level studies report a shared genetic architecture between both disorders [49].

Whether the miRNA changes represent a causal effect or are only markers of a more complex epigenetic model cannot be clarified in our study. It may be interesting to investigate if these miRNA alterations could be transferred into the next generation 
and could increase the risk for cognitive impairment, $A D$ or depression. For depression this transgenerational inheritance could be shown in animal models of mice [4]. Specific miRNA patterns were transferred to the next generations leading to a depressive-like phenotype even if the mice had not been exposed to stressors. Recently a review on paternal exposure to drugs and the transgenerational effect in animal models revealed neuropsychiatric changes including cognition and depressive-like behaviors in future generations [50]. Whether miRNA cause these transgenerational effects needs to be investigated in future studies.

\section{Methodological challenges and limitations}

(1) Although this is the largest miRNA CT study to date, we still believe that in the light of the multifactorial, heterogeneous causes, even larger sample sizes would be desirable. The still relatively small sample size in the clinical sample GANI_MED is due to the limited financial resources. This is also the reason why we filled this gap with an already existing miRNA sample from the general population (SHIP-TREND). But as both studies follow different hypotheses and aims, inclusion/exclusion criteria were different (see refs. $[12,13])$. (2) Another bioinformatics challenge is the high number of miRNA databases which opens the opportunity to search for affected biological systems and pathways. However, this plethora also opens pitfalls, as the assignment of target genes for miRNAs is different, depending on the algorithms and criteria each database applies. Likewise, the identification of significant pathways depending on this assignment is difficult. To partly overcome this issue, we selected more than one database or assignment method and searched for overlap. (3) In our smaller target sample GANI_MED, we accounted for a limited set of covariates. We cannot exclude that observed effects might be due to other covariates like medication effects or other environmental factors which should be addressed in further studies with larger samples. (4) Another methodological issue is the high number of existing miRNAs. According to the Human miRNA tissue Atlas [25] 1997 miRNAs have been detected in 61 tissues. In our screening approach, only 121 miRNAs could be reliably detected, and the relevance of these plasma-circulating miRNAs for brain-related disorders is debatable. Thus, we were only able to catch a glimpse on biological processes and broader approaches including more miRNA data from different tissues might be of value.

Finally, our results shed light on a biological link between childhood trauma and neuropsychiatric outcomes in later life through altered miRNA levels. Whether these miRNA changes are causal or an associated secondary phenomenon which still might be a useful biomarker needs to be investigated in further analyses, preferable in a longitudinal design with $A D$ or depression cases. Nevertheless, we supported our results using bioinformatics and database information from different sources as well as proxy phenotypes from the general population sample. To what extent these alterations in miRNA have the potential to being transferred to the next generation and induce phenotype changes needs to be elucidated in future research.

\section{FUNDING AND DISCLOSURE}

The authors thank Anja Wiechert and Ulrike Lissner for excellent support during sample preparation. SHIP is part of the Community Medicine Research net of the University of Greifswald, Germany, which is funded by the Federal Ministry of Education and Research (grants no. 01ZZ9603, 01ZZ0103, and 01ZZ0403), the Ministry of Cultural Affairs, and the Social Ministry of the Federal State of Mecklenburg-West Pomerania. The Greifswald Approach to Individualized Medicine (GANI_MED) was funded by the Federal Ministry of Education and Research Grant no. 03IS2061A and the German Research Foundation Grant no. GR 1912/5-1. SV was supported by the German Federal Ministry of Education and Research (BMBF) within the framework of the e:Med research and funding concept (Integrament; grant no. 01ZX1614E). HJG has received travel grants and speakers honoraria from Fresenius Medical Care and Janssen Cilag. He has received research funding from the German Research Foundation, the German Federal Ministry of Education and Research (BMBF), the DAMP Foundation, Fresenius Medical Care, the EU "Joint Program Neurodegenerative Disorders" (JPND: 01ED1615), and the European Social Fund (ESF). All other authors state that they have nothing to disclose. All authors declare no competing interests.

\section{ADDITIONAL INFORMATION}

Supplementary Information accompanies this paper at (https://doi.org/10.1038/ s41386-019-0460-2).

Publisher's note: Springer Nature remains neutral with regard to jurisdictional claims in published maps and institutional affiliations.

\section{REFERENCES}

1. Carr CP, Martins CMS, Stingel AM, Lemgruber VB, Juruena MF. The role of early life stress in adult psychiatric disorders: a systematic review according to childhood trauma subtypes. J Nerv Ment Dis. 2013;201:1007-20. https://doi.org/10.1097/ NMD.0000000000000049

2. van der Auwera S, Peyrot WJ, Milaneschi Y, Hertel J, Baune B, Breen G, et al. Genome-wide gene-environment interaction in depression: A systematic evaluation of candidate genes: the childhood trauma working-group of PGC-MDD. Am J Med Genet B Neuropsychiatr Genet. 2018;177:40-9. https://doi.org/10.1002/ ajmg.b.32593

3. Grabe HJ, Wittfeld K, van der Auwera S, Janowitz D, Hegenscheid K, Habes M, et al. Effect of the interaction between childhood abuse and rs 1360780 of the FKBP5 gene on gray matter volume in a general population sample. Hum Brain Mapp. 2016;37:1602-13. https://doi.org/10.1002/hbm.23123

4. Gapp K, Jawaid A, Sarkies P, Bohacek J, Pelczar P, Prados J, et al. Implication of sperm RNAs in transgenerational inheritance of the effects of early trauma in mice. Nat Neurosci. 2014;17:667-9. https://doi.org/10.1038/nn.3695

5. Ramo-Fernández L, Schneider A, Wilker S, Kolassa I-T. Epigenetic alterations associated with war trauma and childhood maltreatment. Behav Sci Law. 2015;33:701-21. https://doi.org/10.1002/bsl.2200

6. Cattane N, Mora C, Lopizzo N, Borsini A, Maj C, Pedrini L, et al. Identification of a miRNAs signature associated with exposure to stress early in life and enhanced vulnerability for schizophrenia: new insights for the key role of miR-125b-1-3p in neurodevelopmental processes. Schizophr Res. 2018. https://doi.org/10.1016/j. schres.2018.07.030

7. Camkurt MA, Güneş S, Coşkun S, Fındıklı E. Peripheral signatures of psychiatric disorders: MicroRNAs. Clin Psychopharmacol Neurosci. 2017;15:313-9. https://doi. org/10.9758/cpn.2017.15.4.313

8. Place RF, Li L-C, Pookot D, Noonan EJ, Dahiya R. MicroRNA-373 induces expression of genes with complementary promoter sequences. Proc Natl Acad Sci USA. 2008;105:1608-13. https://doi.org/10.1073/pnas.0707594105

9. Malan-Müller $\mathrm{S}$, Hemmings SMJ, Seedat $\mathrm{S}$. Big effects of small RNAs: a review of microRNAs in anxiety. Mol Neurobiol. 2013;47:726-39. https://doi.org/10.1007/ s12035-012-8374-6

10. Kumari A, Singh P, Baghel MS, Thakur MK. Social isolation mediated anxiety like behavior is associated with enhanced expression and regulation of BDNF in the female mouse brain. Physiol Behav. 2016;158:34-42. https://doi.org/10.1016/j. physbeh.2016.02.032

11. Dickson DA, Paulus JK, Mensah V, Lem J, Saavedra-Rodriguez L, Gentry A, et al. Reduced levels of miRNAs 449 and 34 in sperm of mice and men exposed to early life stress. Transl Psychiatry. 2018;8:101. https://doi.org/10.1038/s41398-0180146-2

12. Völzke H, Alte D, Schmidt CO, Radke D, Lorbeer R, Friedrich N, et al. Cohort profile: the study of health in Pomerania. Int J Epidemiol. 2011;40:294-307. https://doi. org/10.1093/ije/dyp394

13. Grabe HJ, Assel H, Bahls T, Dörr M, Endlich K, Endlich N, et al. Cohort profile: Greifswald approach to individualized medicine (GANI_MED). J Transl Med. 2014;12:144. https://doi.org/10.1186/1479-5876-12-144

14. Hawley CJ, Gale TM, Smith PSJ, Jain S, Farag A, Kondan R, et al. Equations for converting scores between depression scales (MÅDRS, SRS, PHQ-9 and BDI-II): good statistical, but weak idiographic, validity. Hum Psychopharmacol. 2013;28:544-51. https://doi.org/10.1002/hup.2341

15. Bernstein DP, Stein JA, Newcomb MD, Walker E, Pogge D, Ahluvalia T, et al. Development and validation of a brief screening version of the Childhood Trauma Questionnaire. Child Abus Negl. 2003;27:169-90. 
16. Grabe HJ, Schulz A, Schmidt CO, Appel K, Driessen M, Wingenfeld K, et al. Ein Screeninginstrument für Missbrauch und Vernachlässigung in der Kindheit: der Childhood Trauma Screener (CTS). Psychiatr Prax. 2012;39:109-15. https://doi. org/10.1055/s-0031-1298984

17. Oswald WD, Fleischmann UM. Psychometrics in aging and dementia: advances in geropsychological assessments. Arch Gerontol Geriatr. 1985;4:299-309.

18. Ameling S, Kacprowski T, Chilukoti RK, Malsch C, Liebscher V, Suhre K, et al. Associations of circulating plasma microRNAs with age, body mass index and sex in a population-based study. BMC Med Genom. 2015;8:61. https://doi.org/ 10.1186/s12920-015-0136-7

19. StataCorp. Stata Statistical Software: Release 14. College Station, TX: StataCorp LP; 2015.

20. Vlachos IS, Zagganas K, Paraskevopoulou MD, Georgakilas G, Karagkouni D, Vergoulis $\mathrm{T}$, et al. DIANA-miRPathv3.0: deciphering microRNA function with experimental support. Nucleic Acids Res. 2015;43:W460-6. https://doi.org/ 10.1093/nar/gkv403

21. Wang $\mathrm{X}$. Improving microRNA target prediction by modeling with unambiguously identified microRNA-target pairs from CLIP-ligation studies. Bioinformatics. 2016;32:1316-22. https://doi.org/10.1093/bioinformatics/btw002

22. Hamberg $M$, Backes C, Fehlmann T, Hart M, Meder B, Meese $E$, et al. MiRTargetLink-miRNAs, genes and interaction networks. Int J Mol Sci. 2016;17:564. https://doi.org/10.3390/ijms17040564

23. Vlachos IS, Paraskevopoulou MD, Karagkouni D, Georgakilas G, Vergoulis T, Kanellos I, et al. DIANA-TarBasev7.0: indexing more than half a million experimentally supported miRNA:mRNA interactions. Nucleic Acids Res. 2015;43: D153-9. https://doi.org/10.1093/nar/gku1215

24. Szklarczyk D, Morris JH, Cook H, Kuhn M, Wyder S, Simonovic M, et al. The STRING database in 2017: quality-controlled protein-protein association networks, made broadly accessible. Nucleic Acids Res. 2017;45:D362-8. https://doi.org/10.1093/ nar/gkw937

25. Ludwig N, Leidinger $P$, Becker $K$, Backes $C$, Fehlmann $T$, Pallasch $C$, et al. Distribution of miRNA expression across human tissues. Nucleic Acids Res. 2016:44:3865-77. https://doi.org/10.1093/nar/gkw116

26. Mendes-Silva AP, Pereira KS, Tolentino-Araujo GT, Nicolau EdS, Silva-Ferreira CM, Teixeira AL, et al. Shared biologic pathways between Alzheimer disease and major depression: a systematic review of MicroRNA expression studies. Am J Geriatr Psychiatry. 2016;24:903-12. https://doi.org/10.1016/j.jagp.2016.07.017

27. Chen J, Qi Y, Liu C-F, Lu J-M, Shi J, Shi Y. MicroRNA expression data analysis to identify key miRNAs associated with Alzheimer's disease. J Gene Med. 2018;20: e3014. https://doi.org/10.1002/jgm.3014

28. Chang W-S, Wang Y-H, Zhu X-T, Wu C-J. Genome-wide profiling of miRNA and mRNA expression in Alzheimer's disease. Med Sci Monit. 2017;23:2721-31.

29. Huang F, Long Z, Chen Z, Li J, Hu Z, Qiu R, et al. Investigation of gene regulatory networks associated with autism spectrum disorder based on MiRNA expression in China. PLoS ONE. 2015;10:e0129052. https://doi.org/10.1371/journal.pone.0129052

30. Daimiel-Ruiz L, Klett-Mingo M, Konstantinidou V, Micó V, Aranda JF, García B, et al. Dietary lipids modulate the expression of miR-107, an miRNA that regulates the circadian system. Mol Nutr Food Res. 2015;59:552-65. https://doi.org/10.1002/ mnfr.201400616

31. Scarr E, Craig JM, Cairns MJ, Seo MS, Galati JC, Beveridge NJ, et al. Decreased cortical muscarinic $\mathrm{M} 1$ receptors in schizophrenia are associated with changes in gene promoter methylation, mRNA and gene targeting microRNA. Transl Psychiatry. 2013;3:e230. https://doi.org/10.1038/tp.2013.3

32. Yılmaz ŞG, Erdal ME, Özge AA, Sungur MA. Can peripheral MicroRNA expression data serve as epigenomic (Upstream) biomarkers of Alzheimer's disease? OMICS. 2016;20:456-61. https://doi.org/10.1089/omi.2016.0099

33. Gao B, Hao S, Tian W, Jiang Y, Zhang S, Guo L et al. MicroRNA-107 is downregulated and having tumor suppressive effect in breast cancer by negatively regulating brain-derived neurotrophic factor. J Gene Med. 2017. https://doi.org/ 10.1002/jgm.2932
34. Kumar P, Dezso Z, MacKenzie C, Oestreicher J, Agoulnik S, Byrne M, et al. Circulating miRNA biomarkers for Alzheimer's disease. PLoS ONE. 2013:8:e69807 https://doi.org/10.1371/journal.pone.0069807

35. Ono M, Devilly GJ, Shum DHK. A meta-analytic review of overgeneral memory: the role of trauma history, mood, and the presence of posttraumatic stress disorder. Psychol Trauma. 2016;8:157-64. https://doi.org/10.1037/ tra0000027

36. Cassiers LLM, Sabbe BGC, Schmaal L, Veltman DJ, Penninx BWJH, van den Eede F. Structural and functional brain abnormalities associated with exposure to different childhood trauma subtypes: a systematic review of neuroimaging findings. Front Psychiatry. 2018;9:329. https://doi.org/10.3389/fpsyt.2018.00329

37. Radford K, Delbaere K, Draper B, Mack HA, Daylight G, Cumming R, et al. Childhood stress and adversity is associated with late-life dementia in aboriginal Australians. Am J Geriatr Psychiatry. 2017;25:1097-106. https://doi.org/10.1016/j. jagp.2017.05.008

38. Derkow K, Rössling R, Schipke C, Krüger C, Bauer J, Fähling M, et al. Distinct expression of the neurotoxic microRNA family let-7 in the cerebrospinal fluid of patients with Alzheimer's disease. PLoS ONE. 2018;13:e0200602. https://doi.org/ 10.1371/journal.pone.0200602

39. Hu Y-S, Xin J, Hu Y, Zhang L, Wang J. Analyzing the genes related to Alzheimer's disease via a network and pathway-based approach. Alzheimers Res Ther 2017:9:29. https://doi.org/10.1186/s13195-017-0252-z

40. Daugherty D, Goldberg J, Fischer W, Dargusch R, Maher P, Schubert D. A novel Alzheimer's disease drug candidate targeting inflammation and fatty acid metabolism. Alzheimers Res Ther. 2017;9:50. https://doi.org/10.1186/s13195-0170277-3

41. Estrada LD, Oliveira-Cruz L, Cabrera D. Transforming growth factor beta type I role in neurodegeneration: implications for Alzheimer's disease. Curr Protein Pept Sci. 2018;19:1180-8. https://doi.org/10.2174/1389203719666171129094937

42. Deming Y, Dumitrescu L, Barnes LL, Thambisetty M, Kunkle B, Gifford KA, et al Sex-specific genetic predictors of Alzheimer's disease biomarkers. Acta Neuropathol. 2018. https://doi.org/10.1007/s00401-018-1881-4

43. Gusareva ES, Twizere J-C, Sleegers K, Dourlen P, Abisambra JF, Meier S, et al. Male-specific epistasis between WWC1 and TLN2 genes is associated with Alz heimer's disease. Neurobiol Aging. 2018. https://doi.org/10.1016/j. neurobiolaging.2018.08.001

44. van der Auwera S, Terock J, Teumer A, Schomerus G, Homuth G, Grabe HJ. Gender effects for the interaction of dopamine related genetic variants in BDNF and COMT on memory performance. Pharmacopsychiatry; under review.

45. Hommers L, Raab A, Bohl A, Weber H, Scholz C-J, Erhardt A. et al. MicroRNA hsamiR-4717-5p regulates RGS2 and may be a risk factor for anxiety-related traits. Am J Med Genet B Neuropsychiatr Genet. 2015;168B:296-306. https://doi.org/ 10.1002/ajmg.b.3231

46. Grasso M, Piscopo P, Confaloni A, Denti MA. Circulating miRNAs as biomarkers for neurodegenerative disorders. Molecules. 2014;19:6891-910. https://doi.org/ 10.3390/molecules19056891

47. Mandelli L, Petrelli C, Serretti A. The role of specific early trauma in adult depression: a meta-analysis of published literature. Child trauma adult Depress Eur Psychiatry. 2015;30:665-80. https://doi.org/10.1016/j.eurpsy.2015.04.007

48. Diniz BS, Butters MA, Albert SM, Dew MA, Reynolds CF. Late-life depression and risk of vascular dementia and Alzheimer's disease: systematic review and metaanalysis of community-based cohort studies. Br J Psychiatry. 2013;202:329-35. https://doi.org/10.1192/bjp.bp.112.118307

49. Ye $Q$, Bai $F$, Zhang $Z$. Shared genetic risk factors for late-life depression and Alzheimer's disease. J Alzheimers Dis. 2016;52:1-15. https://doi.org/10.3233/JAD151129

50. Goldberg LR, Gould TJ. Multigenerational and transgenerational effects of paternal exposure to drugs of abuse on behavioral and neural function. Eur $J$ Neurosci. 2018. https://doi.org/10.1111/ejn.14060 